\title{
Kinetically Controlled Growth of Helical and Zigzag Shapes of Carbon Nanotubes
}

\author{
Ruiping Gao, ${ }^{\dagger \neq}$ Zhong L. Wang, ${ }^{*} \dagger$ and Shoushan Fan ${ }^{\S}$ \\ School of Materials Science and Engineering, Georgia Institute of Technology, Atlanta, Georgia 30332-0245, \\ National Natural Science Foundation of China, 35 Huayuan Beilu, East Gate, Beijing 100083, China, and \\ Department of Physics, Tsinghua University, Beijing, 100080 China
}

Received: October 19, 1999; In Final Form: December 6, 1999

\begin{abstract}
This paper focuses on the growth mechanism of the multiwalled carbon nanotubes exhibiting helical and zigzag growth morphologies. Our data support a kinetically controlled growth model in which the creation rates of the pentagon and heptagon carbon rings determine the geometrical shapes of the nanotubes. The carbon nanotube is believed to be grown from a carbon cluster that is nucleated from a pentagon carbon ring followed by a spiral shell growth. The pairing of pentagonal-heptagonal $(\mathrm{P}-\mathrm{H})$ carbon rings is essential in forming the helical and zigzag structures. If there is no twist in the $\mathrm{P}-\mathrm{H}$ orientation along the body of the carbon nanotube, a planar-spiral structure would be formed. If the $\mathrm{P}-\mathrm{H}$ pairs twist their orientations along the growth direction of the nanotube, a helical structure would be formed. The periodicity and the coiling diameter of the helix are determined by the angle of the twist and the distance between the adjacent $\mathrm{P}-\mathrm{H}$ pairs. If the $\mathrm{P}-\mathrm{H}$ pairs are densely accumulated at a local region and the interpair distance is small, a node is formed, thus, the zigzag growth morphology.
\end{abstract}

\section{Introduction}

Multiwalled carbon nanotubes have attracted a great deal of research interests because of their superior mechanical, ${ }^{1}$ electri$\mathrm{cal},{ }^{2}$ chemical, ${ }^{3}$ and many other properties. ${ }^{4}$ Carbon nanotubes exhibit a variety of shapes, such as straight, curved, planarspiral, and helical, simply because of the existence of the hexagon, pentagon, and heptagon carbon rings as a result of its unique hybridization $\mathrm{sp}^{1}, \mathrm{sp}^{2}$, and $\mathrm{sp}^{3}$ bonding. A geometrical combination of the three types of configurations at a different spatial matching and proportion can make any desired geometrical shape. In practice, the fractions of hexagon, pentagon, and heptagon carbon rings being created depend on the experimental conditions, leading to numerous shapes even in the same synthesis process.

The growth of the helical carbon nanotubes was proposed to be determined by the differences between the catalytic activities as well as the diffusion rates of the carbon atoms on the two sides of the catalytic particle, on which the nanotube was nucleated. ${ }^{5}$ This growth mechanism relies on an assumption that the different catalytic activities of the crystallographic facets define the geometry of the carbon nanotube.

We are interested in the geometry and the growth mechanism of the helical carbon nanotubes. After a careful analysis on the helical carbon nanotubes, a new growth mechanism is proposed in this paper that emphasizes the key roles played by kinetics in controlling the shape of the helical nanotubes. A paring of the pentagon and heptagon carbon rings and their orientation determine the geometry of the stretched and the relaxed helical carbon nanotubes. The experimental method is first given in section 2. Following a presentation on the experimental data in section 3 , the growth mechanism and the associated discussion will be given in section 4 .

\footnotetext{
* Corresponding author. E-mail: zhong.wang@mse.gatech.edu.

Georgia Institute of Technology.

$\doteqdot$ National Natural Science Foundation of China.

$\S$ Tsinghua University.
}

\section{Experimental Method}

Carbon nanotubes were produced from a catalytic decomposition of ethylene. The alumna-supported iron catalysts were prepared by putting $\mathrm{Al}_{2} \mathrm{O}_{3}$ fine powders into $\mathrm{Fe}\left(\mathrm{NO}_{3}\right)_{3}$ solution and drying the solution via rotary evaporation and baking. The catalysts were ground into a fine powder. About $10 \mathrm{mg}$ of the catalysts was placed in a ceramic boat and inserted into a quartz tube mounted in a tube furnace. A hydrogen flow $(20 \mathrm{sccm})$, used to reduce the catalyst, was passed through the quartz tube as the furnace was heated to $700{ }^{\circ} \mathrm{C}$. Then, ethylene flow (20 sccm) was introduced and then closed after $20 \mathrm{~min}$. The hydrogen was allowed to flow until the furnace was cooled to room temperature. The valence states of the iron in hydrogen reduced catalysts and grown nanotubes were determined from Mossbauer spectra. The results showed that the iron existed as $\mathrm{Fe}, \mathrm{FeO}$, and $\alpha-\mathrm{Fe}_{2} \mathrm{O}_{3}$ in the hydrogen reduced catalyst, while in the $\mathrm{Fe}-\mathrm{C}$ alloy, $\mathrm{FeO}$, and $\alpha-\mathrm{Fe}_{2} \mathrm{O}_{3}$ after the nanotube growth.

Microstructures of the carbon nanotubes were determined at $400 \mathrm{kV}$ using an JEOL 4000EX high-resolution transmission electron microscope (HRTEM) and at $100 \mathrm{kV}$ using an JEOL 100C TEM. The TEM specimens were prepared by dispersing the as-prepared carbon nanotube in alcohol, then picked up by a carbon film supported by a copper grid.

\section{Experimental Section Results}

Figure 1(parts a and c) shows two TEM images of the helical carbon nanotubes. Part of the nanotube shows the projected coiling shape of the helix, while part displays the stretched zigzag shape with a node at the cross region. The HRTEM image recorded from the zigzag node (Figure 1b) shows that the graphitic layers are circulating around the center of the node and there is a hollow structure at the center. The HRTEM image recorded from the coiling region (Figure 1d) shows the 2-D projection of the coil structure, where only part of the nanotube displays clear graphitic layers at an interplanar distance of $\sim 0.34$ 

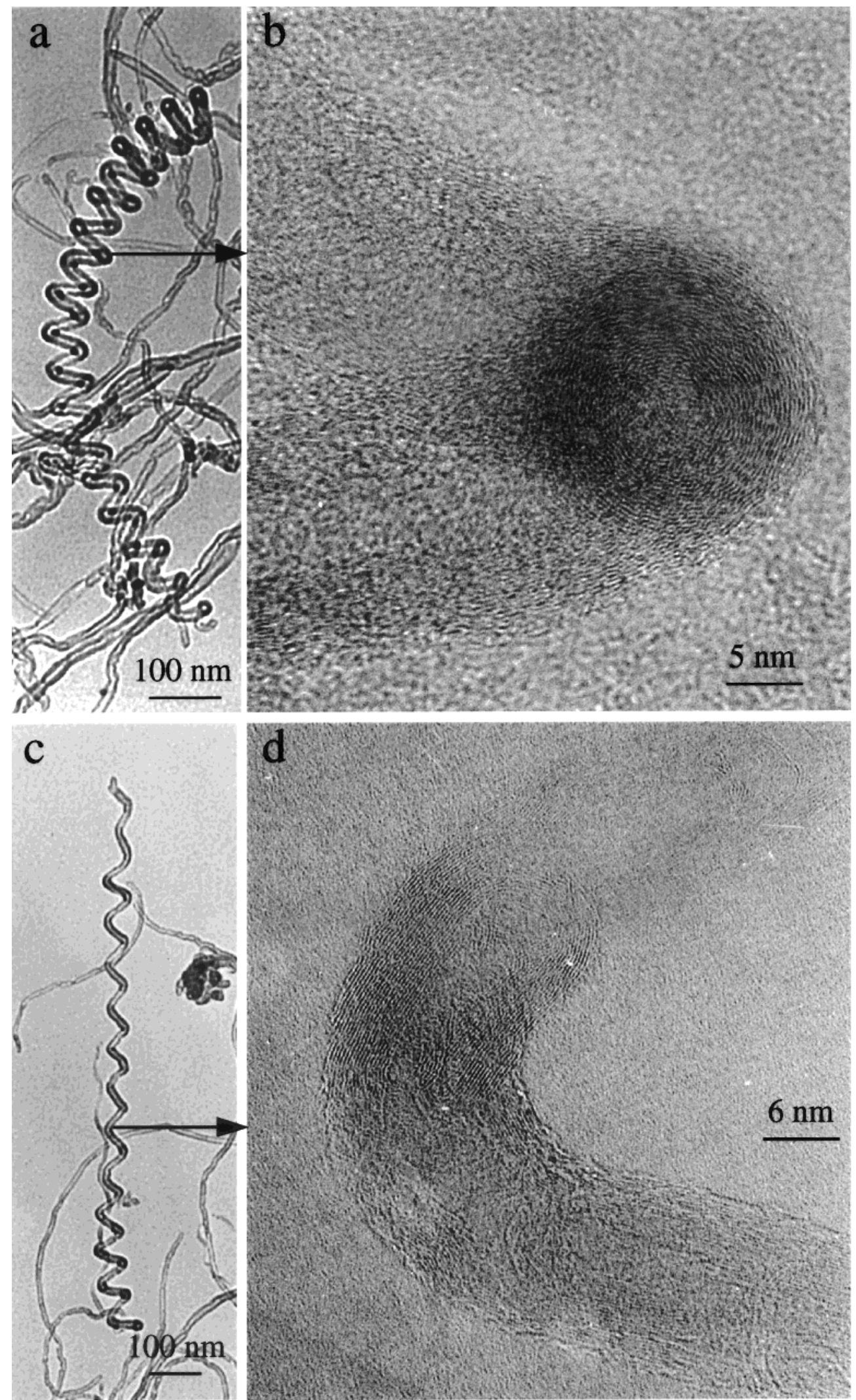

Figure 1. (a, c) Morphologies of the helical and zigzag multiwalled carbon nanotubes. (b, d) High-resolution TEM images recorded from a node at a zigzag region and a coiling region, respectively.

$\mathrm{nm}$, while the remaining part is not clearly resolved because of the 3-D shape of the helix and the limited depth of focus in the TEM (typically $\sim 10 \mathrm{~nm}$ ). The zigzag node and the coil shape are the two typical structures.

To explore the growth mechanism of the helical nanotubes, several questions need to be investigated. One question is whether the nanotubes exhibiting helical structure would have a coiling shape throughout the entire length. Figure $2 \mathrm{a}$ shows a nanotube that exhibits a helical structure at one end while the other end is a curved structure. The periodicity of the coiling also varies along the helical structure. An HRTEM image recorded from the curly region shows the parallel wall structure 

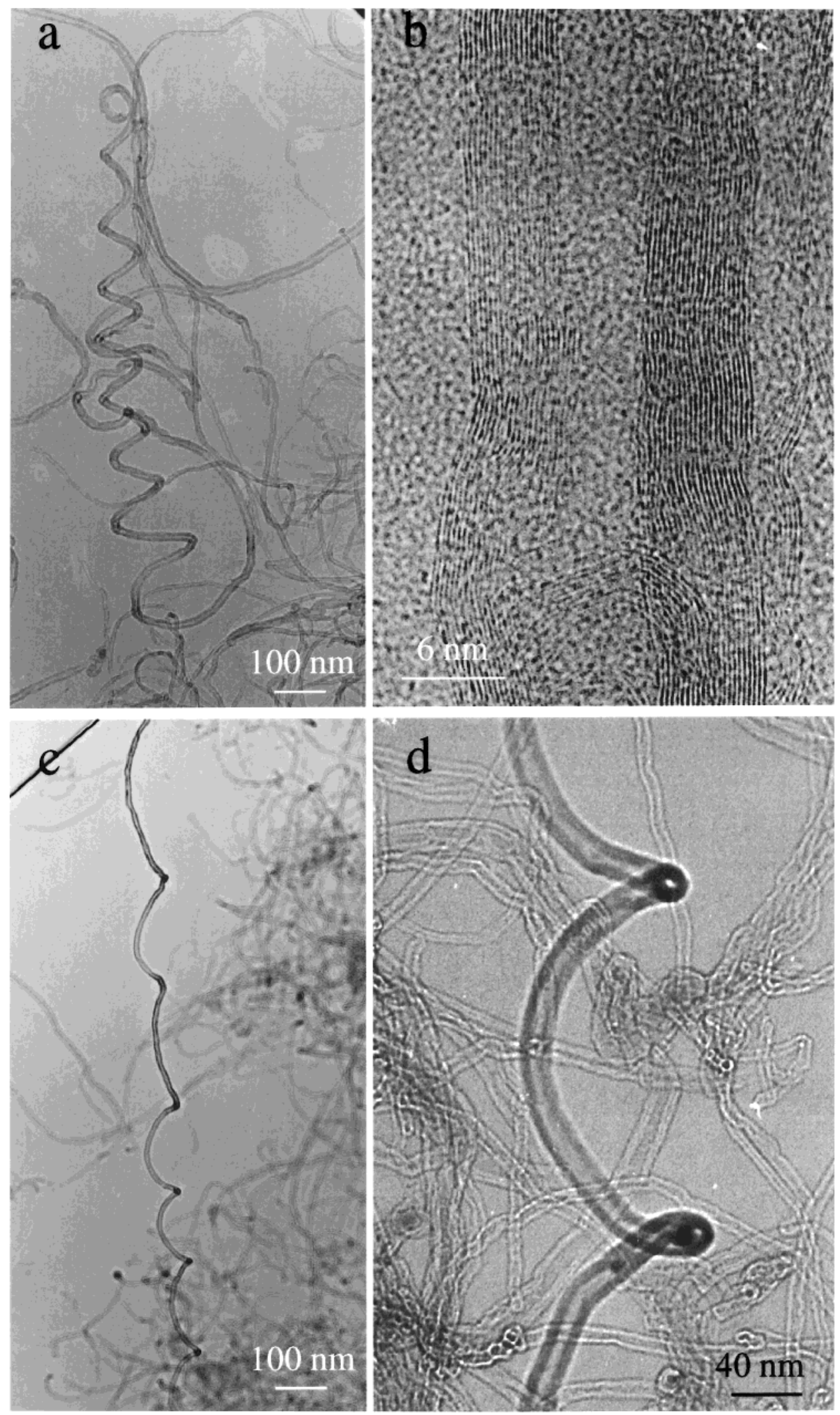

Figure 2. (a, c) Morphology of a helical nanotube with varying diameter and a stretched coiling carbon nanotube. (b) High-resolution TEM image from a straight segment of the carbon nanotube given in (a). (d) A node and a stretched cross point along the body of a carbon nanotube.

of the nanotube (Figure 2b), and the node and a stretched coil are clearly displayed in parts $\mathrm{c}$ and $\mathrm{d}$ of Figure 2. These structures are the growth produced defects.

The second question is about the role played by catalysis in the nucleation and growth of the carbon nanotubes. Figure 3a is a low magnification TEM image recorded from the as- prepared carbon nanotubes. A majority of the nanotubes have a curly shape. The catalytic particles are mixed with the carbon product. Figure 4 shows statistical plots of the size distributions of the catalysis particles and the inner and outer diameters of the carbon nanotubes. The catalysis particles have an average size of $10-12 \mathrm{~nm}$, which is smaller than the average outer 

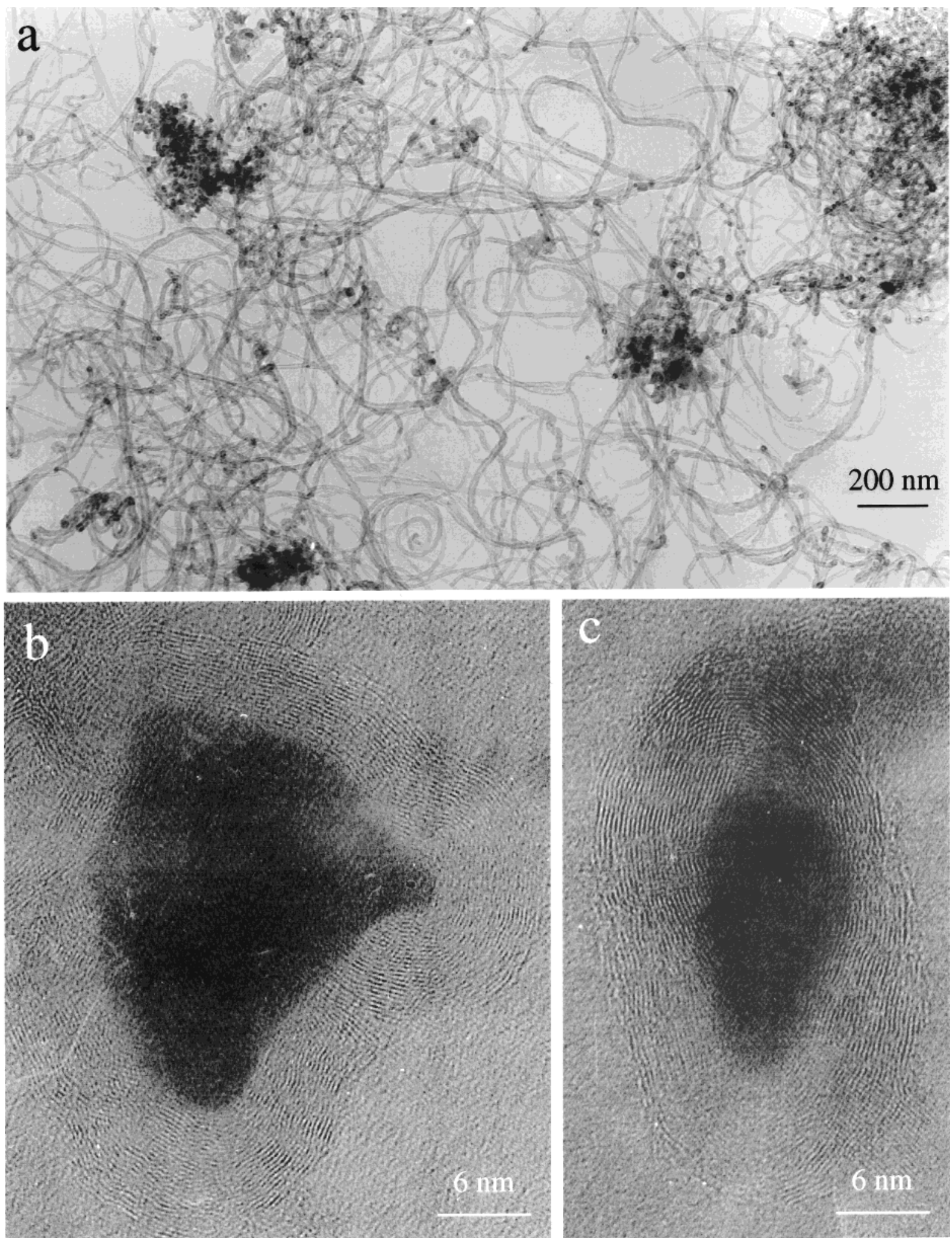

Figure 3. (a) Low magnification TEM image showing the general morphology of the carbon nanotubes and the catalysis particles. (b, c) Typical high-resolution TEM images revealing the encapsulation of the catalysis particles by graphitic shells.

diameter of the carbon nanotubes $(\sim 16-18 \mathrm{~nm})$. Detailed TEM analysis shows that almost all of the catalysis particles are completely encapsulated by carbon. This indicates that the catalysis particles were initially involved in the nucleation of the carbon tubes, but they may not be directly involved in the growth because their catalytic activity had been terminated. A careful search was conducted to see if there are catalysis particles with sizes significantly larger than the diameter of the nanotubes, but we found none. The large size $\mathrm{Al}_{2} \mathrm{O}_{3}$ substrates did not participate in the chemical reaction and they were separated from the carbon nanotubes.

Then a question is whether a nanotube can be nucleated and grown without the presence of a catalysis particle. The planarspiral growth provides an answer to this question (Figure 5a). An HRTEM image recorded from the core of the spiral clearly shows the absence of the catalysis particle (Figure 5b). Another 

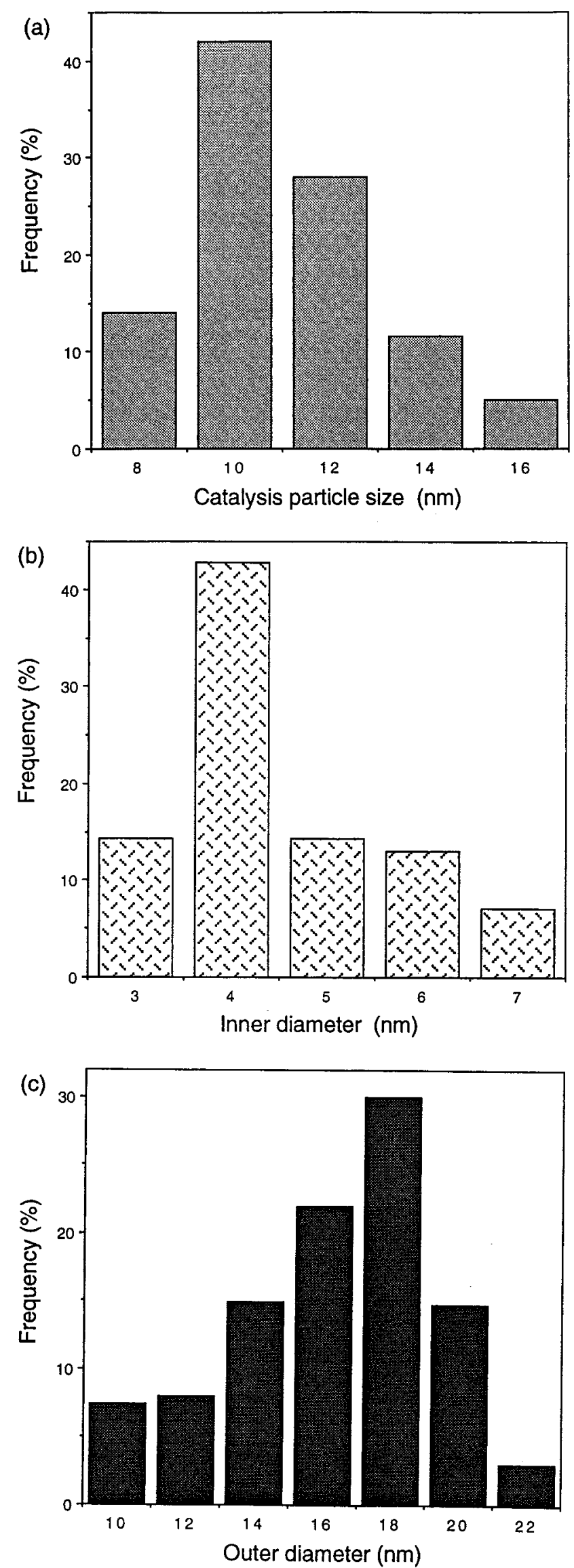

Figure 4. Statistical distributions of (a) the size of the catalysis particles and the (b) inner and (c) outer diameters of the carbon nanotubes.

HRTEM image recorded from the side of the carbon nanotube apparently displays a spiral growth mode of the carbon cluster
(Figure 5c). Therefore, no catalysis particle is mandatory for the nucleation of the carbon structures.

\section{Growth Mechanism}

On the basis of our experimental data presented above, we now examine the growth model of the helical carbon nanotube proposed by Amelinckx et al. ${ }^{5}$ Their growth model would produce the following results. First, there must be catalysis particles whose sizes are significantly larger than the diameters of the carbon nanotubes. These particles are needed to create the bases on which the carbon nanotubes are nucleated and grown, and the helical structure is produced due to the difference in carbon diffusion rates on the crystallographic faces of the particle. Unfortunately, we failed to find these large size particles in our case (Figure 4). The planar-spiral structure has been observed to nucleate and grow without the presence of the catalysis particle at the center. Second, the helical shape of the nanotube should be determined solely by the property of the catalysis particles, if they exist, and the helical structure should be throughout the entire length of the nanotube. But this expectation disagrees with our observation presented in Figure $2 \mathrm{a}$, where a curly part and a coiling part coexist on the same body of the carbon nanotube. Therefore, we believe that the helical structures observed in our specimens are likely to follow a new and different growth mechanism.

The nucleation and growth of carbon structure without a direct contact on the catalyst are possible in several cases. The growth of carbon spheres ${ }^{6}$ and ropes ${ }^{7}$ by a mixed-valent oxide catalytic carbonization process can produce pure carbon products without the enrollment of the catalytic particles in the carbon structure. Carbon spheres with graphitic structure have even been grown without using catalysts. ${ }^{8}$

On the basis of our experimental data, the nucleation and growth of the helical carbon nanotube are proposed as follows. The hexagonal carbon rings are the basic structural units that form the graphite lattice. The pentagonal carbon ring is required to force a hexagonal network to curve inward, forming a surface with positive curvature (Figure 6, parts a and b). The heptagonal carbon ring, on the other hand, makes the hexagonal network curve outward, forming a negative curvature (Figure 6c). ${ }^{9}$ Both pentagonal and heptagonal carbon rings are required to accommodate the change of surface curvature, making it possible, theoretically, to form any geometrical surface. ${ }^{10}$ The pentagon is the start of the spiral-shell growth model (Figure 6d). If a few pentagon carbon rings are introduced in the graphite lattice, a quasi-icosahedral spiral shell can be formed (Figure 6e), but this shell structure is unclosed in order to continuously grow into a larger particle. ${ }^{11}$ This core serves as the base for growing a nanotube.

The continuation of the spiral growth requires a proper supply of a certain fraction of pentagonal carbon-rings in order to accommodate the inward curvature of the spiral core. In practice, however, the nucleation rate of carbon rings, as determined by the experimental conditions, such as temperature and gas-flow rate, may not create the fraction of pentagonal carbon rings required to force the growing layer following the curvature of the inner graphitic layers. If no pentagonal carbon rings are created for a very short period of time and the hexagonal carbon rings are continuously supplied, the spiral layer will grow outward forming a tube structure (Figure 6f), and its subsequent growth would be the same as a straight carbon nanotube. ${ }^{12}$ On the other hand, if there is a catalyst particle encapsulated at the center, spiral growth is also possible (Figure $6 \mathrm{~g}$ ), and the core can be the base for the growth of a carbon nanotube (Figure $6 h)$. 

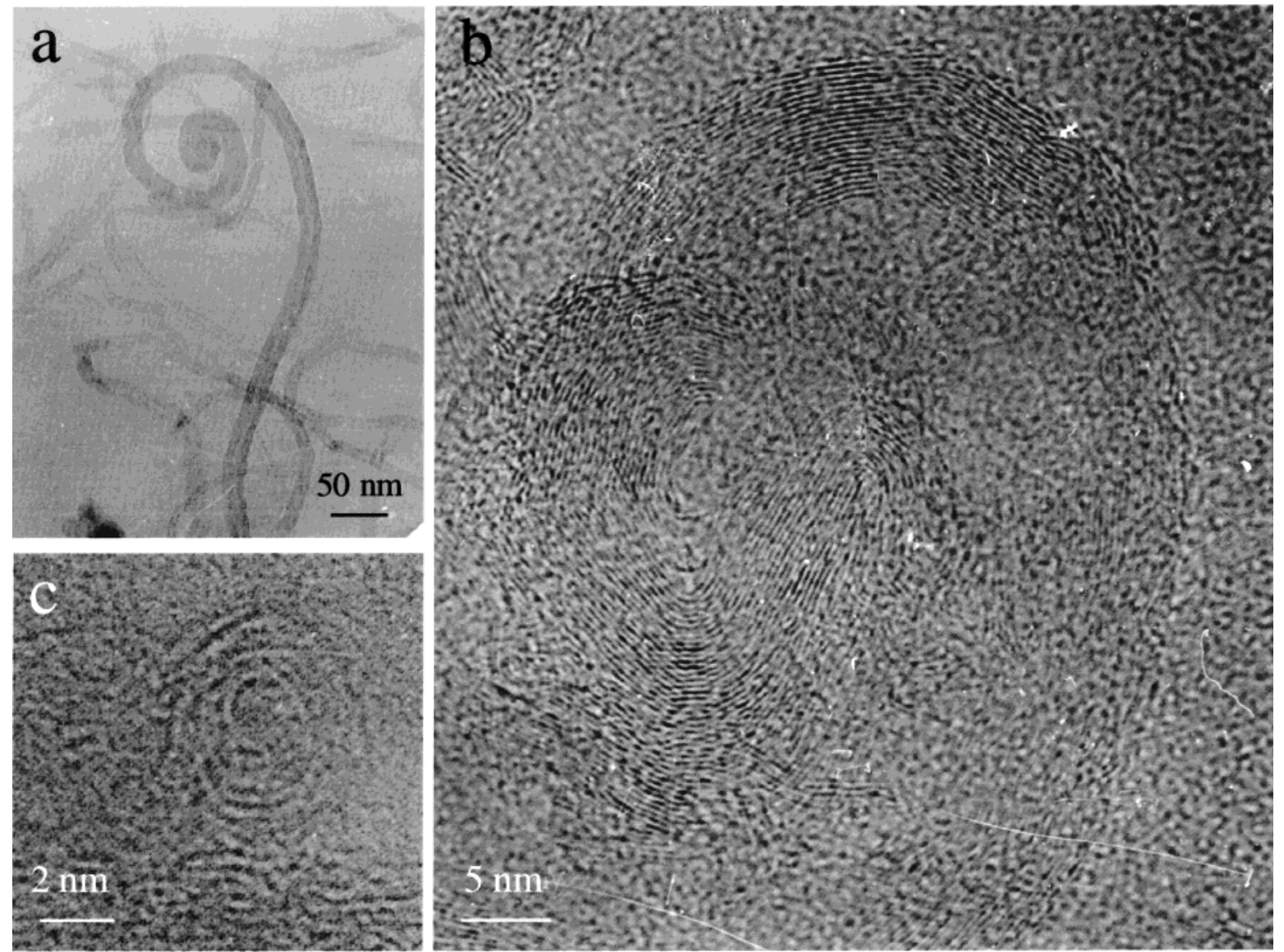

Figure 5. (a) Morphology of a planar-spiral carbon nanotube. (b) High-resolution TEM image recorded from the core of the tube given in (a), showing the absence of the catalysis particles. (c) A carbon cluster formed by a spiral growth without the presence of the catalysis particle at the center.

The formation of the zigzag node and the helical structure is determined by the kinetics during the growth. A common characteristic of the node in a TEM image is a lighter region at the center of the node. The node is produced by introducing the pentagonal and heptagonal $(\mathrm{P}-\mathrm{H})$ pairs followed by a twist, such as the one shown in Figure $2 \mathrm{~d}$, the degree of twist determines the running directions of the two sides of the tube. The formation of the node can be interpreted using the creation of paired $\mathrm{P}-\mathrm{H}$ carbon rings in the hexagonal networks. ${ }^{13}$ The rates at which the pentagons and heptagons are being created determine the morphology of the carbon structure. Previous experimental studies by Kang and Wang ${ }^{6,7}$ have found the eight different geometrical combinations of the hexagon, pentagon, and hexagon carbon rings to fit to any desired geometrical curvature. ${ }^{10,13}$ It was concluded that the $\mathrm{P}-\mathrm{H}$ pairing was the main mechanism for the growth of graphitic carbon spheres and carbon ropes. ${ }^{6,7}$ Geometrical modeling by Fonseca et al. ${ }^{14}$ also agrees with this result.

To illustrate the growth of helical structure in the current experiments, only three parallel graphitic shells of the carbon tube are schematically shown in Figure 7a. If a pair of pentagonal and heptagonal carbon rings is created, the tube will be forced to turn, for example, clockwise. Another turn is introduced if a second pair of $\mathrm{P}-\mathrm{H}$ carbon ring is introduced. The number of $\mathrm{P}-\mathrm{H}$ pairs determines the total twist angle. The graphitic layers grown near the core of the twist still follow the shape and curvature of the inner tube. As more graphitic layers are built up, a node close to a spherical shape is formed. ${ }^{6}$

The creation of the paired pentagonal and heptagonal carbon rings is a key factor in producing various shapes of graphitic layers. From the formation of nodes along the tubes, it can be suggested that the pairs of pentagonal and heptagonal carbon rings are not created continuously throughout the entire process but periodically, possibly resulting in the periodically distributed nodes as well as the helical structure. The geometrical shape of the tube is determined by the fractions and creation rates of pentagonal, hexagonal, and heptagonal carbon rings. This is a kinetically controlled growth process.

The growth of the coil structure is also associated with the presence of the $\mathrm{P}-\mathrm{H}$ pairs. The bending of the tube is equivalent to a faster growth on the pentagon side due to positive curvature (stretching) and a slower growth on the heptagon side due to the negative curvature (compression); thus, a planar-spiral structure can be produced if the $\mathrm{P}-\mathrm{H}$ pairs are oriented in the same plane. ${ }^{14}$ Alternatively, a helical tube can be grown if the orientations of the $\mathrm{P}-\mathrm{H}$ pairs rotate along the body of the tube. Such a model was previously proposed by Ihara and Itoh $^{15}$ in interpretation of the structure of a helically coiled $\mathrm{C}_{360}$. An extension of the model and the relevant restrictions for multiwalled helical structure has been given by Setton and Setton. ${ }^{16}$ The twisting of the $\mathrm{P}-\mathrm{H}$ pairs along a coiling nanotube is schematically illustrated in Figure $7 \mathrm{~b}$. The periodicity $(L)$ and the coiling diameter $(D)$ of the helix are determined by the twist angle and the distance between the adjacent $\mathrm{P}-\mathrm{H}$ pairs, which can vary in practice, resulting in the change in coiling periodicity and the diameters of the coils (see Figure 2a). The growth of the helical structure is continuous if the $\mathrm{P}-\mathrm{H}$ pairs are continuously produced; otherwise, a straight section of the tube can be grown if the $\mathrm{P}-\mathrm{H}$ pairs are absent, in agreement with the result shown in Figures 1a and 2a. In practice, the fluctuation 


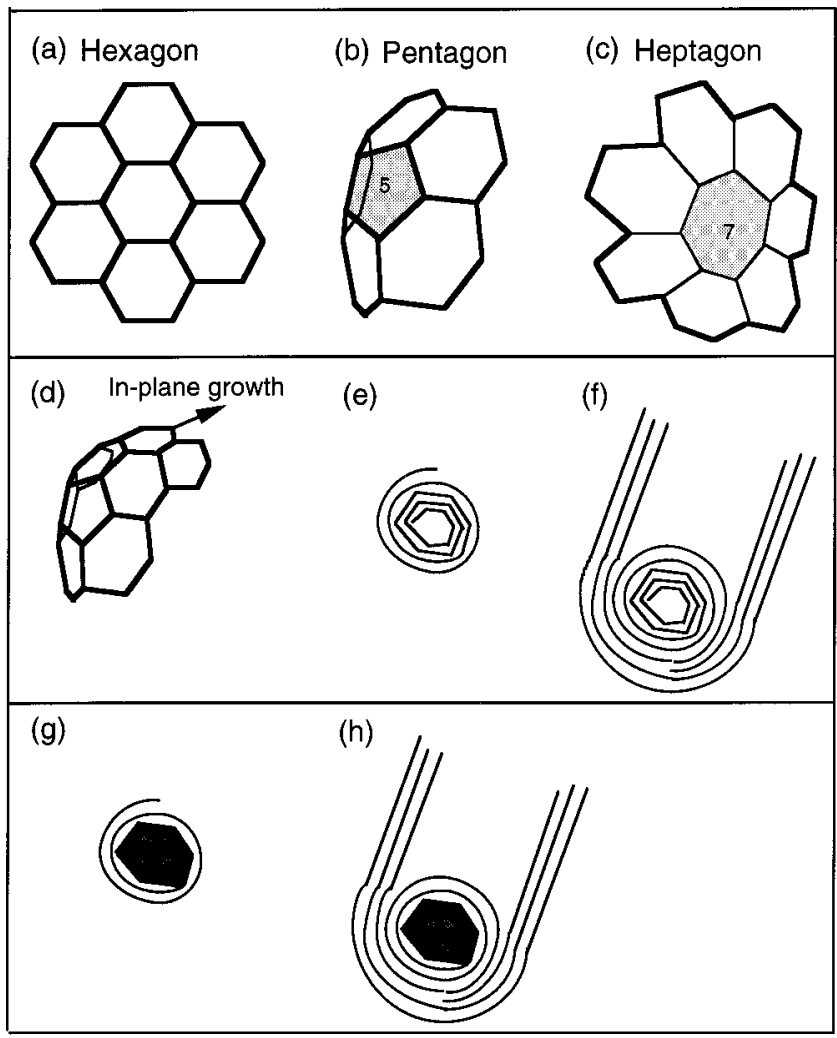

Figure 6. $(\mathrm{a}-\mathrm{c})$ Hexagon, pentagon, and heptagon carbon rings in the matrix of the graphitic layer. (d) The spiral growth initiated from a pentagon carbon-ring. (e) A carbon cluster formed by the spiral-shell growth. (f) A carbon nanotube grown from the carbon cluster. (g) A carbon encapsulated catalysis particle. (h) Growth of carbon nanotube from the encapsulated particle.

(a)
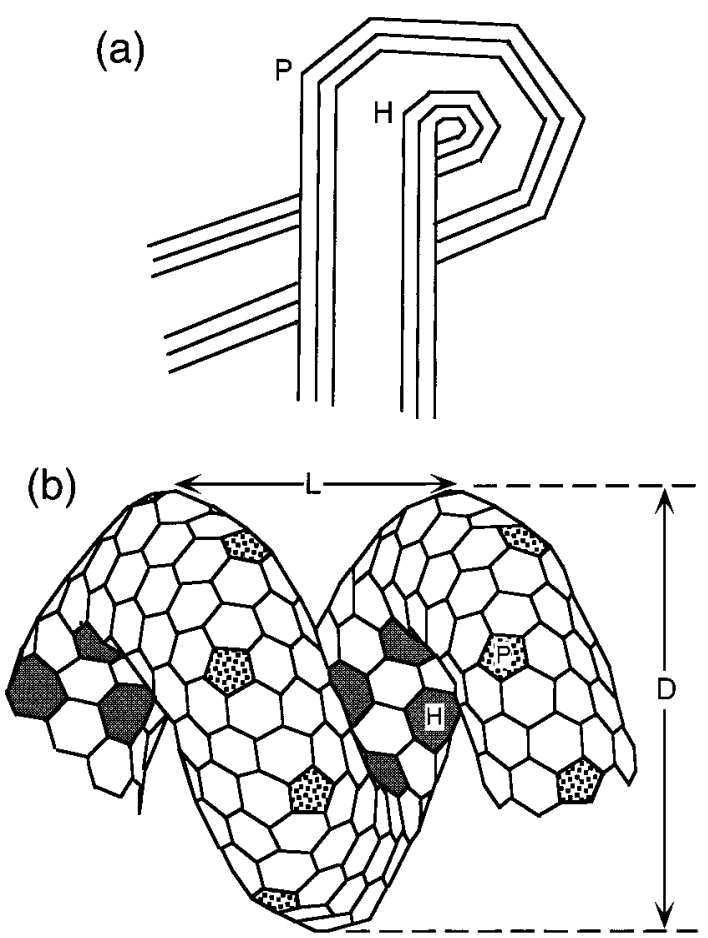

Figure 7. (a) Formation of a node along the body of a carbon nanotube as a result of introducing the paired pentagon $(\mathrm{P})$ and heptagon $(\mathrm{H})$ carbon rings. The node is formed if the density of the $\mathrm{P}-\mathrm{H}$ pairs is high and the interpair distance is short. (b) Formation of a helical carbon nanotube by twisting the orientations of the $\mathrm{P}-\mathrm{H}$ pairs along the body of the nanotube. A coil structure is formed if the twisting angle and interpair distance are kept constant. in the creation rates of the $\mathrm{P}-\mathrm{H}$ pairs can produce a complex shape. This is likely to be the reason that the structures of carbon are very versatile.

The model presented in Figure $7 \mathrm{~b}$ is only for a single walled carbon nanotube. For a multiwalled helical nanotube, the formation of $\mathrm{P}-\mathrm{H}$ pairs may not need to be synchronized through the thickness for the following reason. TEM of multiwalled carbon nanotube has found that the spiral angle of the graphitic layer varies from layer to layer. Thus, there is little correlation between the structures of individual layers as along as the interlayer distance is kept at $\sim 0.34 \mathrm{~nm}$, hence no correlation in the distribution of $\mathrm{P}-\mathrm{H}$ pairs through the thickness.

\section{Conclusions}

In this paper, the growth mechanism of the helical and zigzag carbon nanotubes is examined using the information provided by high-resolution transmission electron microscopy. We found that the catalytic particles may not be directly encapsulated at the nucleation cores of the nanotubes and the periodicity and coiling diameter vary along the body of the nanotube. More importantly, the size of the catalytic particles is smaller than the exterior diameter of the nanotubes, and no large size catalytic particles were found onto which the carbon nanotubes would attach. Our data support kinetically controlled growth of the helical nanotube.

The carbon nanotube is believed to be grown from a carbon cluster (or node) that is nucleated from a pentagon carbon ring followed by a spiral shell growth. The creation rates of the pentagon and heptagon carbon rings determine the geometrical shape of the nanotube. It is proposed that the pairing of pentagonal-heptagonal $(\mathrm{P}-\mathrm{H})$ carbon-rings is essential in forming the helical and zigzag structures. If there is no twist on the $\mathrm{P}-\mathrm{H}$ orientation along the body of the carbon nanotube, a planar-spiral structure would be formed. If the $\mathrm{P}-\mathrm{H}$ pairs twist their orientations along the growth direction of the nanotube, a helical structure would be formed. The periodicity and the coiling diameters of the helix are determined by the twist angle and the distance between the adjacent $\mathrm{P}-\mathrm{H}$ pairs. If the $\mathrm{P}-\mathrm{H}$ pairs are densely accumulated at a local region and the interpair distance is small, a node is formed, thus, the zigzag growth morphology. A straight tube would be grown if the pentagon and heptagon carbon rings are absent.

Acknowledgment. We are grateful for the assistance from Jinsong Yin, Yongqian Wang, and Zhigang Bai. Research was sponsored by the National Natural Science Foundation of China and NSF Grants CHE-9727633 and DMR-9733160. Thanks to the Georgia Tech Electron Microscopy Center for providing the experimental facility.

\section{References and Notes}

(1) See, for example: Poncharal, P.; Wang, Z. L.; Ugarte, D.; de Heer, W. A. Science 1999, 283, 1513. de Heer, W. A.; Chatelain, A.; Ugarte, D. Science 1995, 17, 1179.

(2) See, for example: Frank, S.; Poncharal, P.; Wang, Z. L.; de Heer, W. A. Science 1998, 280, 1744.

(3) Chen, J.; Hamon, M. A.; Hu, H.; Chen, Y.; Rao, A. M.; Eklund, P. C.; Haddon, R. C. Science 1998, 282, 95. Dillon, A. C.; Jones, K. M.; Bekkedahl, T. A.; Kiang, C. H.; Bethuune, D. S.; Heben, M. J. Nature 1997, 386, 377.

(4) Saito, R.; Dresselhaus, G.; Dresselhaus, M. S. Physical Properties of Carbon Nanotubes; Imperial College Press: London, 1998.

(5) Amelinckx, S.; Zhang, X. B.; Bernaerts, D.; Zhang, X. F.; Ivanov, V.; Nagy, J. B. Science 1994, 265, 635.

(6) Kang, Z. C.; Wang, Z. L. Philos. Mag. 1996, B73, 905. 
(7) Wang, Z. L.; Kang, Z. C. Philos. Mag. 1996, B74, 51.

(8) Govindaraj, A.; Sen, R.; Nayaraju, B. V.; Rao, C. N. R. Philos. Mag. Lett. 1997, 363, 367.

(9) Iijima, S.; Ichihashi, T.; Ando, Y. Nature 1992, 356, 776.

(10) Ösawa, E.; Yoshida, M.; Fujita, M. MRS Bulletin XIX 33-36 (November, 1994).

(11) Kroto, H. W.; McKay, K. Nature 1988 331, 328.
(12) Iijima, S.; Ajayan, P. M.; Ichihashi, T. Phys. Rev. Lett. 1992, 69, 3100.

(13) Kang, Z. C.; Wang, Z. L. J. Phys. Chem. B 1996, 100, 17725.

(14) Fonseca, A.; Hernadi, K.; Nagy, J. B.; Lambin, Ph.; Lucas, A. A Carbon 1995, 33, 1759.

(15) Ihara, S.; Itoh, S. Carbon 1995, 33, 931.

(16) Settton, R.; Setton, N. Carbon 1997, 33, 497. 